\title{
Vibration performances of MRE embedded sandwich beam: experimental study
}

\author{
Biswajit Nayak ${ }^{1}$, Raj Kishor Das ${ }^{2}$, Satyam Shivam Gautam ${ }^{3}$ \\ ${ }^{1}$ GITA Bhubaneswar, Bhubaneswar, Odisha, India \\ ${ }^{2}$ Raghu Engineering College, Visakhapatnam, Andhra Pradesh, India \\ ${ }^{2,3}$ North Eastern Regional Institute of Science and Technology, Nirjuli, Arunachal Pradesh, India \\ ${ }^{1}$ Corresponding author \\ E-mail: ${ }^{1}$ biswajitgy@gmail.com, ${ }^{2}$ drrkdviit@gmail.com, ${ }^{3}$ satyam.gautam@gmail.com
}

Received 12 November 2018; accepted 19 November 2018

DOI https://doi.org/10.21595/vp.2018.20383

Check for updates

Copyright $\mathbb{C} 2018$ Biswajit Nayak, et al. This is an open access article distributed under the Creative Commons Attribution License, which permits unrestricted use, distribution, and reproduction in any medium, provided the original work is properly cited.

\begin{abstract}
In this paper, magnetorheological elastomer (MRE) is fabricated with carbonyl iron particle mixed with silicon rubber as the matrix. Sandwiched beam is fabricated from the MRE sandwiched between two aluminum layers. Forced vibrations of the MRE cored sandwiched beam have been studied. An experimental set up is fabricated to investigate the vibration responses of MRE cored sandwich beam with and without magnetic field. The obtained experimental results reveal that the natural frequencies and damping ratios of the MRE cored sandwiched beams are significantly influenced by MRE and magnetic field. The stiffness and damping properties of the beam change due to the change in rheological properties of the MRE under the magnetic field.
\end{abstract}

Keywords: isotropic MRE, forced vibration, carbon black, magnetic field.

\section{Introduction}

Magnetorheological (MR) materials are smart materials whose rheological properties such as viscosity and shear modulus response rapidly to the external stimulus i.e. the magnetic field intensities. Magnetorheological elastomers (MREs) typically consist of magnetically polarisable particles in a non-magnetic elastomer such as synthetic or natural rubber. Particles inside the non-magnetic elastomer can be distributed homogeneously or may be combined to form a chain like columnar structures under the application of steady state magnetic field [1-3]. MREs are being used in semi-active or active vibration devices for different applications. Some of the devices are bushing withvariable stiffness suspension, tunable spring elements, adaptive tuned vibration absorber, tunable automotive mounts and structures [4-6].

Various factors such as volume percentage, particle size, elastomer matrices as well as the structural alignment of the particles under a magnetic field influence the rheological properties of the MRE [7-9]. Maximum magnetorheological effect on the elastomers can be obtained when the magnetic particle fill rate is around $30 \%$ of the entire volume [8,9]. Various matrix materials such as natural rubber and thermoplastic elastomers and also new methods have been used to fabricate new MREs $[10,11]$. To enhance the mechanical performance of the MREs, Chen et al. [10] used the carbon black along with iron particles in the non-magnetic elastomer.

Mostly, the works on mechanical performances of isotropic MREs considers the combinations of different synthetic and natural rubbers and iron particles [12-14]. Zajac et al. [12] fabricated the isotropic magnetorheological elastomers considering iron powder and the thermoplastic matrix as the filling materials. The experimentally investigation of Rao et al. [13] shows the functional behavior of magnetorheological gels under static and dynamic shear conditions in magnetic field environment. Lu et al. [14] used the carbonyl iron (CI) particles with a poly (styrene-ethylenebutylene-styrene) tri-block co-polymer which is used as the thermoplastic rubber matrix to fabricate a novel smart thermoplastic magnetorheological elastomer composite.

For last few decades the MR materials have been used as core materials to obtain controllable properties of sandwich beams under magnetic field [15-17]. It was shown that the vibration of a sandwich beam can be minimized by embedding MR materials in the sandwich beam. In recent 
years, the development of MRE embedded sandwich beams was initiated. The flexural rigidities of MRE cored sandwich beams are controlled with magnetic field due to field dependent shear modulus of the MRE core. The dynamic flexural rigidity change of MRE embedded soft cored sandwiched beams with conductive and nonconductive skins under the application of magnetic field based on higher order theory was investigated by Zhou and Wang [18, 19]. Wei et al. [20] experimentally obtained the natural frequencies and responses of the MRE cored sandwich beam with magnetic field and excitation frequencies. The vibration characteristics of an MRE cored sandwich beam subjected to non-homogeneous magnetic field were presented experimentally by $\mathrm{Hu}$ et al. [21]. It was shown that the sandwiched beam has the capability to left shift the first natural frequency when the magnetic field is increased in the activated regions. The dynamic analysis of an MRE embedded viscoelastic cored sandwich beam subjected to periodic axial load for various resonance cases have been investigated by Nayak et al. [22, 23]. However, it is observed that the research on the experimental work of the effect of various system parameters of sandwich beam such as skin thickness, core thickness and isotropic MREs with and without carbon black on dynamic characteristics is still lacking.

In this work, the silicon rubber based isotropic MRE is fabricated. The MRE embedded sandwich beams with aluminum skins have been fabricated. A set up has been made incorporating permanent magnetic field with provision for varying the air gap between the magnet and specimen so as to change the magnetic field. The forced vibration analysis of the MRE embedded sandwich beam is done to study the dynamic behaviors.

\section{Preparation of MRE and sandwich beams}

To fabricate isotropic MREs, three material components, including particles, matrix and additives are used. The particles used are carbonyl iron powder (BASF, Germany) obtained from supplier Vimal Intertrade Private Limited. The particle is fine, hard powder (mechanically) with size distributions viz. $\mathrm{d} 10=1.5 \mu \mathrm{m}, \mathrm{d} 50=5 \mu \mathrm{m}$ and $\mathrm{d} 90=10 \mu \mathrm{m}$. The vulcanized (RTV) silicone (at room temperature), catalyst (CAT-13219, Shin-Etsu Chemical Co. Ltd.) are supplied by Swastika Constant Care, Kolkata.

During fabrication of MRE, first the carbonyl iron powders are homogeneously mixed along with RTV and catalyst. Then the mixture is stirred for approximately 45 minutes till even dispersion is achieved. The mixture is kept in the vacuum chamber to remove air bubbles. Then the mixture is poured into aluminum moulds. After the air bubbles are completely removed from the mixture, the mixture is then poured into the aluminum mould. The RTV silicone is mixed to liquid catalyst for which the ratio is 10:1. The compositions of the MRE sample are presented in Table 1.

Table 1. The composition of each MRE prepared

\begin{tabular}{|c|c|}
\hline \multicolumn{2}{|c|}{ Composition $(V \%)$} \\
\hline Carbonyl iron powder & RTV silicone and catalyst mixture \\
\hline 40 & 60 \\
\hline
\end{tabular}

The sandwich beam is fabricated with aluminum skins and MRE core. The thickness of fabricated MRE used in the sandwich beam is $1.8 \mathrm{~mm}$. Also, the thickness of aluminum skin used for the skin is $1.28 \mathrm{~mm}$. Due to low damping characteristics and relatively high stiffness of the aluminum compared to that of MRE, the aluminum is chosen for the skin material. The length and width of the sandwich beams are $245 \mathrm{~mm}$ and $20 \mathrm{~mm}$ respectively.

\section{Experimentation}

To study the forced vibration of the sandwich beam with and without magnetic field an experimental set up is fabricated as shown in Fig. 1. In this experiment, the MRE sandwich beam was clamped to a fixed platform using a cantilever configuration. The experimental set up is 
integrated with permanent magnets, sensor, shaker, PC driven ACE dynamic signal analyzer and signal analysis equipments. The instruments used in the experiment include Bruel and Kjaer made PULSE Lab Shop, Rotational Laser Vibrometer, shaker and amplifier. A magnetic field is generated by permanent magnets over the test sandwich beam. The magnetic field is applied in the vertical direction i.e. perpendicular to the surface of the skins. In this experiment the maximum field intensity generated by the permanent magnets is $1000 \mathrm{G}$. Changing the air gap between the sandwich beam and the permanent magnets varies the magnetic field. The tip of the shaker is connected to the sandwich beam at the actuation location of $80 \mathrm{~mm}$ from the fixed end of the beam. The laser point is located at the tip of the sandwich beam.

The shaker is driven by the voltage signal from the power amplifier. This voltage signal is generated by acquisition system output through PULSE Lab Shop. The laser sensor is used to measure vibration displacement at a single location, and this sensor could sense the velocity from 0 to $200 \mathrm{~mm} / \mathrm{s} / \mathrm{V}$. The software, PULSE Lab Shop is used for obtaining and analyzing the acquired analogue signals from laser sensor through data acquisition. The Tesla gauge provided by Scientific Equipment and Services is used to measure the amplitude of strength of magnetic field.

To excite the sandwich beam, different options were employed such as impact hammer and shaker. In each case, even though the applied forces are different, and the sandwich beam vibrated at different amplitudes, the beam vibrated at the same natural frequency. In a modal test the shaker is used to excite the sandwich beam.

The experimental procedure is summarized as follows; first the free vibration of the sandwich beam was studied. Then the forced vibration was studied. The PULSE Lab Shop is set using a swept sine actuation at a range of $0-300 \mathrm{~Hz}$ with $1 \mathrm{~Hz}$ increment. The voltage amplitude of the excitation signal is set at $200 \mathrm{mV}$. The signal output from the computer is send to the shaker through the amplifier. Shaker provides the external vibration over the test sandwich beam. The laser sensor acquires the vibration data for the test sandwich beam, which are then sent to the computer through controller and data acquisition system.

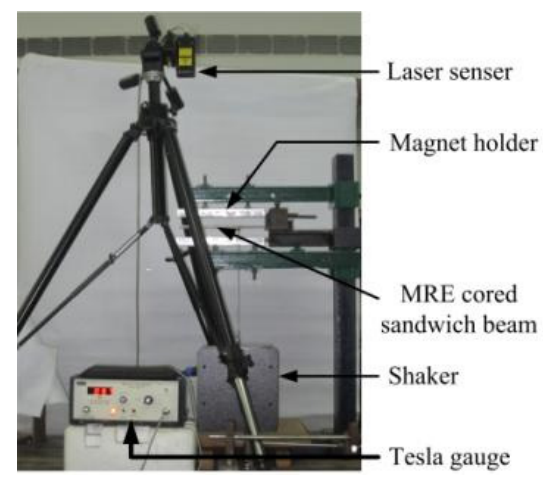

Fig. 1. Experimental set-up

The input signal is processed in PULSE Lab Shop software in the computer. The outputs viz. natural frequencies, vibration response and vibration amplitudes are presented in the results for analysis.

\section{Results and discussion}

The response of the vibration frequency of a structure subjected to an external dynamic force depends on the distribution of mass as well as the stiffness of structural elements. Likewise, the vibration magnitude is controlled by the damping behaviors of structure. Hence, the vibration behaviors of the magnetorheological elastomer embedded sandwich beams under different magnetic field amplitudes were studied with the help of their resonant natural frequencies.

Fig. 2 shows the vibration response of the sandwich beam with MRE core for the first three 
modes. In this test, the distances between the magnetic poles are varied to apply the required magnetic field intensities. The frequencies obtained from the experiment are presented in the Tables 2. It is observed that the modal frequencies of the sandwiched-beam increased as the magnetic field are increased. This shows that the trend of a frequency is towards right shift.

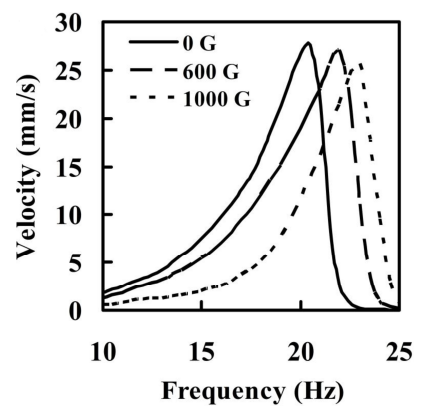

a)

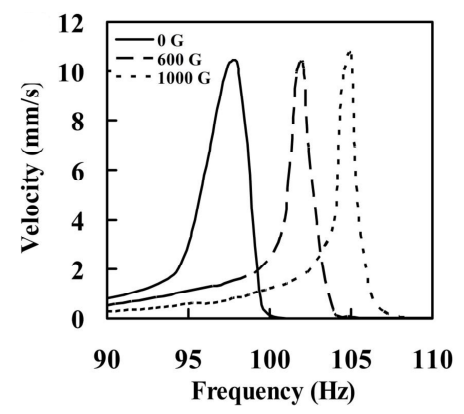

b)

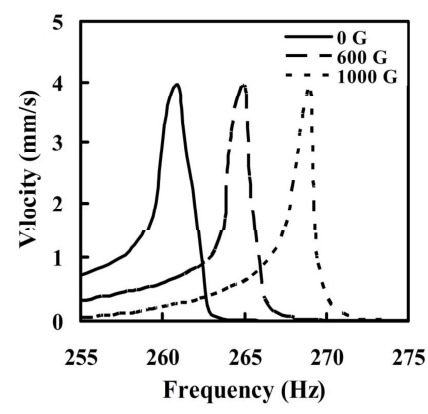

c)

Fig. 2. Vibration responses of MRE cored sandwiched-beam (1.28 $\mathrm{mm}$ skin and $1.8 \mathrm{~mm}$ skin thickness) under different magnetic field: a) mode 1, b) mode 2 , c) mode 3

The frequencies of sandwich beam with different MRE core are presented in Table 2. It can be observed that the modal frequencies of sandwich beam increase with an increase in magnetic field. It is due to the rise in shear modulus of the MRE in magnetic field condition.

Table 2. Modal frequencies obtained from experiment and its comparison with the frequencies obtained from analytical and FE methods for the initial three modes of a sandwiched-beam under different magnetic field. (Beam of $1.28 \mathrm{~mm}$ skin and $1.8 \mathrm{~mm}$ core and MRE without carbon black)

\begin{tabular}{|c|c|c|c|c|c|c|c|c|}
\hline \multicolumn{3}{|c|}{ 0 G } & \multicolumn{3}{c|}{$600 \mathrm{G}$} & \multicolumn{3}{c|}{$1000 \mathrm{G}$} \\
\hline Mode 1 & Mode 2 & Mode 3 & Mode 1 & Mode 2 & Mode 3 & Mode 1 & Mode 2 & Mode 3 \\
\hline 20.50 & 98.00 & 261.00 & 22.00 & 102.00 & 265.00 & 23.00 & 105.00 & 269.00 \\
\hline
\end{tabular}

Fig. 3 shows the time responses for forced vibration of MRE cored sandwiched-beam to an impulse applied at free end in the absence and presence of a magnetic field $(600 \mathrm{G})$.

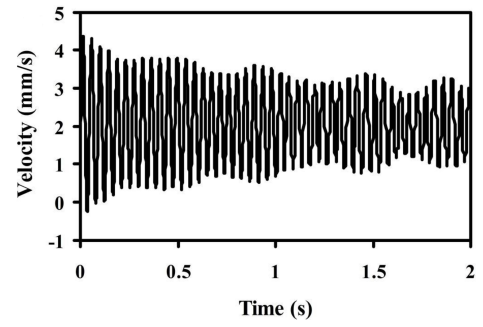

a)

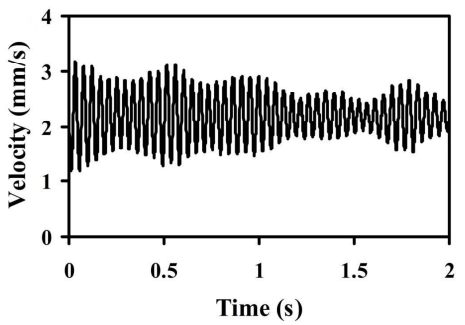

b)

Fig. 3. Vibration responses of MRE (without carbon black) cored sandwich beam (1.28 mm skin thickness and $1.8 \mathrm{~mm}$ core thickness): a) without magnetic field, b) with magnetic field of $600 \mathrm{G}$

The data shows that the damping of the beam increases significantly with an applied magnetic field. The decay rate of the response in the plots of forced vibration in terms of damping ratio $(\zeta)$ can be explained as follows:

$$
\begin{aligned}
& \delta=\frac{1}{n} \ln \frac{x_{1}}{x_{n+1}}, \\
& \zeta=\frac{\delta}{2 \pi},
\end{aligned}
$$


where $\delta, x, n$ corresponds to log decrement, vibration amplitude and number of cycles respectively. In case of sandwiched-beam with MRE core, the damping ratio equals 0.0031 without magnetic field and is increased to 0.0055 for a magnetic field of $600 \mathrm{G}$. It results a $43.64 \%$ increase in damping ratio.

\section{Conclusions}

The isotropic silicon rubber based MRE is fabricated. For the vibration analysis the MRE embedded sandwich beam with aluminum skins has been fabricated. A set up has been also fabricated incorporating permanent magnetic field with provision for varying the air gap between the magnet and specimen so as to change the magnetic field. The magnetic field in the static condition has been applied here.

From this experimental study of MRE cored sandwiched-beam it has been observed that the natural frequency is found to be increased with an increase in magnetic field. The rise in magnetic field increases the shear modulus of the MRE causing the shifting of natural frequency due to the change in stiffness of the system. The logarithmic decrement method has been used for finding the damping of the system including the magnetic field effect. The damping ratio is found to be increased from 0.0031 to 0.0055 for MRE embedded sandwich beam.

\section{References}

[1] Chen L., Gong X. L., Li W. H. Microstructures and viscoelastic properties of anisotropic magnetorheological elastomer. Smart Materials and Structures, Vol. 16, 2007, p. 2645-2650.

[2] Gong X. L., Zhang X. Z., Zhang P. Q. Fabrication and characterization of isotropic magnetorheological elastomers. Polymer Testing, Vol. 24, 2005, p. 669-676.

[3] Chen L., Gong X. L., Jiang W. Q., Yao J. J., Deng H. X., Li W. H. Investigation on magnetorheological elastomers based on natural rubber. Journal of Material Science, Vol. 42, 2007, p. 5483-5489.

[4] Watson J. R. Method and Apparatus for Varying the Stiffness of a Suspension Bushing. US Patent No. 5609353, 1997.

[5] Kallio M. The Elastic and Damping Properties of Magnetorheological Elastomers. Ph.D. Thesis, Tampere University of Technology, 2005.

[6] Sun S., Deng H., Yang J., Li W., Du H., Alici G., Nakano M. An adaptive tuned vibration absorber based on multilayered MR elastomers. Smart Materials and Structures, Vol. 24, 2015, p. 045045.

[7] Jolly M. R., Bender J. W., Carlson J. D. Properties and applications of commercial magnetorheological fluids. Journal of Intelligent Material Systems and Structures, Vol. 10, Issue 1, 1999, p. 5-13.

[8] Davis L. C. Model of magnetorheological elastomers. Journal of Applied Physics, Vol. 85, 1999, p. $3348-3351$.

[9] Lokander M., Stenberg B. Performance of isotropic magnetorheological rubber materials. Polymer Testing, Vol. 22, Issue 3, 2003, p. 245-251.

[10] Chen L., Gong X. L., Li W. H. Effect of carbon black on the mechanical performances of magnetorheological elastomers. Journal of Polymer Testing, Vol. 27, 2008, p. 340-345.

[11] Kaleta J., Krolewicz M., Lewandowski D. Magnetomechanical properties of anisotropic and isotropic magnetorheological composites with thermoplastic elastomer matrices. Smart Materials and Structures, Vol. 20, 2011, p. 085006.

[12] Zajac P., Kaleta J., Lewandowski D., Gasperowicz A. Isotropic magnetorheological elastomers with thermoplastic matrices: structure, damping properties and testing. Smart Materials and Structures, Vol. 19, 2010, p. 045014.

[13] Rao P. V., Maniprakash S., Srinivasan S. M., Srinivasa A. R. Functional behavior of isotropic magnetorheological gels. Smart Materials and Structures, Vol. 19, 2010, p. 085019.

[14] Lu X., Qiao X., Watanabe H., Gong X. L., Yang T., Li W., Sun K., Li M., Yang K., Xie H., Yin Q., Wang D., Chen X. Mechanical and structural investigation of isotropic and anisotropic thermoplastic magnetorheological elastomer composites based on poly (styrene-b-ethylene-cobutylene-b-styrene) (SEBS). Rheogica Acta, Vol. 51, 2012, p. 37-50. 
[15] Sun Q., Zhou J.-X., Zhang L. An adaptive beam model and dynamic characteristics of magnetorheological materials. Journal of Sound and Vibration, Vol. 261, 2003, p. 465-481.

[16] Cantera M. A., Behrooz M., Gibson R. F., Gordaninejad F. Modeling of magneto-mechanical response of magnetorheological elastomers (MRE) and MRE-based systems: a review. Smart Materials and Structures, Vol. 26, 2017, p. 020401.

[17] Rajamohan V., Rakheja S., Sedaghati R. Vibration analysis of a partially treated multi-layer beam with magnetorheological fluid. Journal of Sound and Vibration, Vol. 329, 2010, p. 3451-3469.

[18] Zhou G. Y., Wang Q. Use of magnetorheological elastomer in an adaptive sandwich beam with conductive skins. Part I: magnetoelastic loads in conductive skins. International Journal of Solids and Structures, Vol. 43, 2006, p. 5386-5402.

[19] Zhou G. Y., Wang Q. Use of magnetorheological elastomer in an adaptive sandwich beam with conductive skins. Part II: dynamic property. International Journal of Solids and Structures, Vol. 43, 2006, p. 5403-5420.

[20] Wei K.-X., Meng G., Zhang W.-M., Zhu S.-S. Experimental investigation on vibration characteristics of sandwich beams with magnetorheological elastomers cores. Journal of Central South University of Technology, Vol. 15, 2008, p. 239-242.

[21] Hu G., Guo M., Li W., Du Alici H. G. Experimental investigation of the vibration characteristics of a magnetorheological elastomer sandwich beam under non-homogeneous small magnetic fields. Smart Materials and Structures, Vol. 20, 2011, p. 127001.

[22] Nayak B., Dwivedy S. K., Murthy K. S. R. K. Dynamic analysis of magnetorheological elastomerbased sandwich beam with conductive skins under various boundary conditions. Journal of Sound and Vibration, Vol. 330, 2011, p. 1837-1859.

[23] Nayak B., Dwivedy S. K., Murthy K. S. R. K. Multi-frequency excitation of magnetorheological elastomer-based sandwich beam with conductive skins. International Journal of Non-Linear Mechanics, Vol. 47, 2012, p. 448-460. 